\title{
COINCIDENCE AND FIXED POINT RESULTS FOR NON-COMMUTING MAPS
}

\author{
ABDUL LATIF AND SALEH A. AL-MEZEL
}

Abstract. In the setting of Banach spaces, some results on the existence of coincidence and common fixed points for single-valued and multivalued non-commuting maps with and without contractive type conditions are obtained.

\section{Introduction and preliminaries}

Let $(X, d)$ be a metric space. We denote $C B(X)$ for the family of nonempty closed bounded subsets of $X$, and $H$ for the Hausdorff metric on $C B(X)$, i.e., for any $A, B \in C B(X)$,

$$
H(A, B)=\max \left\{\sup _{a \in A} d(a, B), \sup _{b \in B} d(b, A)\right\}
$$

where $d(a, B)=\inf \{d(a, b): b \in B\}$ is the distance from the point $a$ to the subset $B$.

Let $M$ be a nonempty subset of a metric space $X$. Let $T: M \rightarrow C B(M)$ be a multivalued map and $f: M \rightarrow M$ a continuous single-valued map.

i) $T$ is called an $f$-contraction [2] if there exists a constant $h \in(0,1)$ such that for all $x, y \in K$,

$$
H(T(x), T(y)) \leq h d(f(x), f(y))
$$

ii) $T$ is called a generalized $f$-contraction $[1,4]$ if there exists a constant $h \in(0,1)$ such that for all $x, y \in M$,

$$
\begin{aligned}
H(T(x), T(y)) \leq & h \max \{d(f(x), f(y)), d(f(x), T(x)), d(f(y), T(y)), \\
& \left.\frac{1}{2}[d(f(x), T(y))+d(f(y), T(x))]\right\}
\end{aligned}
$$

If $M$ is a nonempty closed subset of a Banach space $X$. Then

iii) $T$ and $f$ are compatible [4] if $f T(x) \in C B(M)$ for each $x \in M$ and $\lim _{n \rightarrow \infty} H\left(T\left(f\left(x_{n}\right)\right)\right.$, $\left.f\left(T\left(x_{n}\right)\right)\right)=0$ whenever $\left\{x_{n}\right\}$ is a sequence in $M$ such that $\lim _{n \rightarrow \infty} T x_{n}=K \in C B(M)$ and $\lim _{n \rightarrow \infty} f x_{n}=t \in K$.

Received September 21, 2006.

2000 Mathematics Subject Classification. 47H10, 54H25.

Key words and phrases. Fixed point, coincidence point, metric space, Banach space, R-weakly commuting maps, R-subweakly commuting maps, $f$-contraction, generalized $f$-contraction. 
iv) $T$ and $f$ are reciprocally continuous [11] on $M$ if $f T(x) \in C B(M)$ for each $x \in M$ and $\lim _{n \rightarrow \infty} f T x_{n}=f K, \lim _{n \rightarrow \infty} T f x_{n}=T t$ whenever $\left\{x_{n}\right\}$ is a sequence in $M$ such that $\lim _{n \rightarrow \infty} T x_{n}=$ $K \in C B(M)$ and $\lim _{n \rightarrow \infty} f x_{n}=t \in K$.

v) $T$ and $f$ are $R$-weakly commuting [9], if there exists a positive constant $R$ such that, for each $x \in M$

$$
H(T(f(x)), f(T(x))) \leq R\|f(x)-T(x)\| .
$$

vi) $M$ is called $q$-starshaped if $q \in M$ and $\lambda x+(1-\lambda) q \in M$ for all $x \in M$ and all $\lambda$ with $0 \leq \lambda \leq 1$.

vii) For a $q$-starshaped set $M$, define for all $x \in M$ and for all $\lambda_{n}, 0 \leq \lambda_{n} \leq 1$

$$
J_{n}(x)=\lambda_{n} T(x)+\left(1-\lambda_{n}\right) q .
$$

Note that, for each $n \geq 1, J_{n}$ carries $M$ into $C B(M)$.

For a $q$-starshaped shaped set $M$ we say

viii) $T$ and $f$ are $R$-subcommuting [8] if for each $x \in M, f T(x) \in C B(M)$ and there exists a positive constant $R$ such that for $f(q)=q$, and $0<\lambda \leq 1$

$$
H(T(f(x)), f(T(x))) \leq \frac{R}{\lambda}\left\|f(x)-J_{\lambda}(x)\right\|
$$

ix) $T$ and $f$ are $R$-subweakly commuting [10] if for each $x \in M, f T(x) \in C B(M)$ and there exists a positive constant $R$ such that for $f(q)=q$ and $0 \leq \lambda \leq 1$,

$$
H(T(f(x)), f(T(x))) \leq R\left\|f(x)-J_{\lambda}(x)\right\|,
$$

where, $J_{\lambda}(x)=\lambda T(x)+(1-\lambda) q$.

Note that each commuting map implies $R$-subcommuting, which implies $R$-weakly commuting but the converse of these implications are not true [8]. Also, commuting maps are $R$-subweakly commuting but not conversely [10].

A point $x \in M$ is called a fixed point of $T$ if $x \in T(x)$ and the set of fixed points of $T$ is denoted by Fix $(T)$. A point $x \in M$ is called a coincidence point of $f$ and $T$ if $f(x) \in T(x)$. We denote by $C(f \cap T)$ the set of coincidence points of $f$ and $T$.

For the setting of single-valued and multivalued maps, Kaneko [2, 3] proved coincidence and common fixed point results for commuting and weakly commuting maps. In [4], Kaneko and Sessa have introduced the notion of compatible maps and proved a coincidence point result for such maps. Pathak [7] has given the concept of weakly compatible maps and generalized the coincidence point result of Kaneko and Sessa[4]. In fact, commuting maps are weakly commuting, weakly commuting are compatible and compatible are weakly compatible but the converse implication are not true in general. On the other hand, in the setting of metric and Banach spaces, Latif and Abu-Hajar [5], Latif and Tweddle [6] have proved some coincidence and common fixed point results for commuting maps. Recently, Rhoades [8] has generalized these results by introducing the notion of $R$-subcommuting maps. While, Shahzad[10] introduced a notion $R$-subweakly commuting maps and studied the coincidence and common fixed point results of [6] under this concept. 
In this paper, we prove some new results on the existence of coincidence and common fixed points for $R$-subweakly commuting maps in the setting of Banach spaces.

To make the paper self-contained, we recall the following results due of Kaneko and Sessa [4],

Theorem 1.1. Let $(X, d)$ be a complete metric space, $f: X \rightarrow X$ and $T: X \rightarrow C B(X)$ be compatible continuous maps such that $T(X) \subseteq f(X)$ and $T$ is a generalized $f$-contraction. Then $C(f \cap T) \neq \varnothing$.

Lemma 1.2. Let $(X, d)$ be a complete metric space, $f: X \rightarrow X$ and $T: X \rightarrow C B(X)$ be compatible maps. Then $f T(z)=T f(z)$ provided $z \in C(f \cap T)$.

\section{Main results}

First we prove our results on the existence of coincidence points.

Theorem 2.1. Let $X$ be a Banach space and let $M$ be a nonempty closed q-star-shaped subset of $X$. Let $f: M \rightarrow M$ be a continuous affine map such that $f(M)=M$ and $f(q)=q$. Let $T: M \rightarrow C B(M)$ be a multivalued map which $R$-subweakly commutes with $f$. Suppose that each of the maps $J_{n}$ is continuous generalized $f$-contraction. Then

a) $C\left(f \cap J_{n}\right) \neq \varnothing$, for any $n \geq 1$.

b) $C(f \cap T) \neq \varnothing$, provided $T(M)$ is bounded and $(f-T) M$ is closed.

Proof. a) Choose a sequence $\left\{h_{n}\right\}$ of a real numbers with $0<h_{n}<1$ and $h_{n} \rightarrow 1$ as $n \rightarrow$ $\infty$. Since $M$ is a closed subset of a Banach space $X$, then $M$ is a complete metric space and $T(M) \subset M=f(M)$. Also, for each $n, J_{n}(M) \subset M=f(M)$. Now we show that for any $n \geq 1, J_{n}$ and $f$ are compatible maps. Let $\left\{y_{n}\right\} \subset M$ be such that for a fixed $\alpha, J_{\alpha}\left(y_{n}\right) \rightarrow K \in C B(M)$ and $f\left(y_{n}\right) \rightarrow y \in K$, as $n \rightarrow \infty$. Then, since $f$ is affine and $R$-subweakly commutes with $T$ we get,

$$
\begin{aligned}
H\left(J_{\alpha} f\left(y_{n}\right), f J_{\alpha}\left(y_{n}\right)\right) & =\left\|h_{n} T f\left(y_{n}\right)+\left(1-h_{n}\right) q-h_{n} f T\left(y_{n}\right)-\left(1-h_{n}\right) q\right\| \\
& =h_{n} H\left(T f\left(y_{n}\right), f T\left(y_{n}\right) \leq h_{n} R\left\|f\left(y_{n}\right)-J_{\alpha}\left(y_{n}\right)\right\| .\right.
\end{aligned}
$$

Thus, $H\left(J_{\alpha} f\left(y_{n}\right), f J_{\alpha}\left(y_{n}\right)\right) \rightarrow 0$ as $n \rightarrow \infty$ and hence for any $n \geq 1, J_{n}$ and $f$ are compatible maps. Therefore, by Theorem 1.1 $C\left(f \cap J_{n}\right) \neq \varnothing$, for any $n \geq 1$.

b) From the proof of part (a), there exists an $x_{n} \in M$ such that $f\left(x_{n}\right) \in J_{n}\left(x_{n}\right)=h_{n} T\left(x_{n}\right)+(1-$ $\left.h_{n}\right) q$, and so there exists some $w_{n} \in T\left(x_{n}\right)$ such that $f\left(x_{n}\right)=h_{n} w_{n}+\left(1-h_{n}\right) q$. Thus,

$$
\left\|f\left(x_{n}\right)-w_{n}\right\|=\left(1-h_{n}\right)\left\|q-w_{n}\right\| .
$$

Since $T(M)$ is bounded and $w_{n} \in T\left(x_{n}\right) \subset T(M)$, we have that $\left\|q-w_{n}\right\|$ is bounded, and so by the fact $h_{n} \rightarrow 1$ and $n \rightarrow \infty$, it follows that $\left(f\left(x_{n}\right)-w_{n}\right) \rightarrow 0$ as $n \rightarrow \infty$. Now, since

$$
f\left(x_{n}\right)-w_{n} \in f\left(x_{n}\right)-T\left(x_{n}\right)=(f-T)\left(x_{n}\right) \subset(f-T) M,
$$

and $(f-T) M$ is closed, we get $0 \in(f-T) M$ and thus $C(f \cap T) \neq \varnothing$. 
Theorem 2.2. Let $X$ be a Banach space and let $M$ be a nonempty closed $q$-star-shaped subset of $X$. Let $f: M \rightarrow M$ be a continuous affine map such that $f(M)=M$ and $f(q)=q$. Let $T: M \rightarrow C B(M)$ be a multivalued map which $R$-subweakly commutes with $f$. Suppose that each of the maps $J_{n}$ is reciprocally continuous with respect to $f$. Then

a) $C\left(f \cap J_{n}\right) \neq \varnothing$, for any $n \geq 1$.

b) $C(f \cap T) \neq \varnothing$, provided $T(M)$ bounded and $(f-T) M$ closed.

Proof. a) Following the proof of Theorem 2.1(a), for a fixed but arbitrary natural number $\alpha$ we get $H\left(J_{\alpha} f\left(y_{n}\right), f J_{\alpha}\left(y_{n}\right)\right) \rightarrow 0$ as $n \rightarrow \infty$, where $J_{\alpha}\left(y_{n}\right) \rightarrow K \in C B(M)$ and $f\left(y_{n}\right) \rightarrow y \in K$, as $n \rightarrow \infty$. Now, since the maps $J_{\alpha}$ and $f$ are reciprocally continuous, we get $H\left(J_{\alpha}(y), f(K)\right)=0$ and $J_{\alpha}(y)=f(K)$. Since $y \in K, f(y) \in f(K)$ and $f(y) \in J_{\alpha}(y)$. Therefore $C\left(f \cap J_{n}\right) \neq \varnothing$, for any $n \geq 1$.

b) From part (a), there exists $x_{n} \in M$ such that $f\left(x_{n}\right) \in J_{n}\left(x_{n}\right)=h_{n} T\left(x_{n}\right)+\left(1-h_{n}\right) q$, and the rest of the proof same as the proof of Theorem 2.1(b).

Now we prove some common fixed point results.

Theorem 2.3. Assume that either all the hypotheses of Theorem 2.1 or Theorem 2.2 hold. If $f\left(z_{n}\right) \neq f^{2}\left(z_{n}\right)$ implies $z_{n} \notin C\left(f \cap J_{n}\right)$, then Fix $\left(J_{n}\right) \cap$ Fix $(f) \neq \varnothing$ for any $n \geq 1$.

Proof. Suppose that all the hypotheses of Theorem 2.1 (or Theorem 2.2) hold. Then for each $n \geq 1$ there exists $z_{n} \in M$ such that $f\left(z_{n}\right) \in J_{n}\left(z_{n}\right)$. It follows from the proof of Theorem 2.1 part (a) that each of the $J_{n}$ and $f$ are compatible. So applying Lemma 1.2, we have

$$
f\left(z_{n}\right)=f\left(f\left(z_{n}\right)\right) \in f J_{n}(z)=J_{n} f(z),
$$

and thus $f\left(z_{n}\right)$ is a common fixed point of $J_{n}$ and $f$.

Lemma 2.4. Let $X$ be a Banach space and let $M$ be a nonempty $q$-starshaped subset of $X$. Let $f: M \rightarrow M$ and $T: M \rightarrow C B(M)$ be R-subweakly commuting maps such that $f(q)=q$. Then $f T(z)=T f(z)$ provided $z \in C\left(f \cap J_{\lambda}\right)$.

Proof. If $z \in C\left(f \cap J_{\lambda}\right)$, then by the $R$-subweak commutativity of $f$ and $T$ we have

$$
H(T(f(z)), f(T(z))) \leq R\left\|f(z)-J_{\lambda}(z)\right\|=0 .
$$

Thus, we get $f(T(z))=T(f(z))$.

Applying Lemma 2.4 we have the following common fixed point result.

Theorem 2.5. Let $X$ be a Banach space and let $M$ be a nonempty $q$-starshaped subset of $X$. Let $f: M \rightarrow M$ and $T: M \rightarrow C B(M)$ be R-subweakly commuting maps such that $f(q)=q$. If there exists a point $z \in M$ such that $f^{2}(z)=f(z) \in J_{\lambda}(z) \cap T(z)$, then Fix $(T) \cap F i x(f) \neq \varnothing$.

Proof. Applying Lemma 2.4, we have

$$
f(z)=f(f(z)) \in f T(z)=T f(z)
$$


which implies that $f(z)$ is a common fixed point of $T$ and $f$.

\section{Acknowledgement}

The authors thank the referee for his/her suggestions and comments.

\section{References}

[1] P. Z. Daffer and H. Kaneko, Fixed points generalized contractive multi-valued mappings, J. Math. Anal. and Appl. 192(1995), 655-666.

[2] H. Kaneko, Single-valued and multivalued f-contractions, Boll. Un. Mat. Ital. 6(1985), 29-33.

[3] H. Kaneko, A common fixed point of weakly commuting multi-valued mappings, Math. Japonica 33(1988), 741-744.

[4] H. Kaneko and S. Sessa, Fixed point theorems for compatible multivalued and single-valued mappings, Internat. J. Math. \& Math. Sci. 12(1989), 257-262.

[5] A. Latif and A. Abu-Hajar, On coincidence and hybrid fixed point, Radovi Matematicki 11(2002), 119-123.

[6] A. Latif and I. Tweddle, On multivalued f-nonexpancive maps, Demon. Math. 36(1999), 564-574.

[7] H. K. Pathak, Fixed point theorems for weak compatible multivalued and single-valued mapping, Act. Math. Hungar. 76(1995), 69-78.

[8] B. E. Rhoades, On multivalued f-nonexpansive maps, Fixed Point Theory and Appl. 2(2001), 89-92.

[9] N. Shahzad and T. Kamran, Coincidence points and R-weakly commuting maps, Arch. Math. (Brno) 37(2001), 179-183.

[10] N. Shahzad, Coincidence points and R-subweakly commuting multivalued maps, Demonstratio Mathematica 36(2003), 427-431.

[11] S. L. Singh and S. N. Mishra, Coincidence and fixed points of reciprocally continuous and compatible hybrid maps, Inter. J. Math \& Math. Sci. 30(2002), 627-635.

Department of Mathematics, King Abdul Aziz University, P.O.Box 80203, Jeddah-21589, Saudi Arabia.

E-mail:latifmath@yahoo.com

Department of Mathematics, King Abdul Aziz University, P.O.Box 80203, Jeddah-21589, Saudi Arabia.

E-mail: mathsaleh@yahoo.com 